\title{
Research on the influence of fog on laser transmission based on Monte Carlo method (Withdrawal Notice)
}

Z. Tan, Y. C. Gong, X. Zhang, Y. T. Yang, K. Leng

Z. Tan, Y. C. Gong, X. Zhang, Y. T. Yang, K. Leng, "Research on the influence of fog on laser transmission based on Monte Carlo method (Withdrawal Notice)," Proc. SPIE 11562, AOPC 2020: Advanced Laser Technology and Application, 1156209 (5 November 2020); doi: 10.1117/12.2575592

SPIE Event: Applied Optics and Photonics China (AOPC 2020), 2020, Beijing, China 


\title{
Research on the influence of fog on laser transmission based on Monte Carlo method (Withdrawal Notice)
}

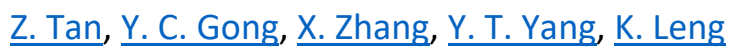

Author Affiliations -

Z. Tan, ${ }^{1}$ Y. C. Gong, ${ }^{1}$ X. Zhang, ${ }^{1}$ Y. T. Yang, ${ }^{1}$ K. Leng ${ }^{1}$

${ }^{1}$ Army Engineering Univ. (China)

Proceedings Volume 11562, AOPC 2020: Advanced Laser Technology and Application; 1156209

(2020) https://doi.org/10.1117/12.2575592

Event: Applied Optics and Photonics China (AOPC 2020), 2020, Beijing, China

Online publication date: 5 November 2020

Withdrawn from publication: 1 December 2020

Publisher's Note: This paper, originally published 5 November 2020, was withdrawn per author request. 